\title{
INOCULAÇÃO DE Bacillus subtilis E Azospirillum brasilense NA CULTURA DO MILHO
}

Rita de Cássia Lima Mazzuchelli ${ }^{1}$; Bruno Ferrari Sossai ${ }^{2}$; Fabio Fernando de Araujo ${ }^{3}$

Universidade do Oeste Paulista - UNOESTE, ${ }^{1,3}$ Programa de Pós Graduação em Agronomia, ${ }^{2,3}$ Especialização em Integração lavoura pecuária floresta, Presidente Prudente - SP. E-mail: bfs sossai@yahoo.com.br , ritamazzuchelli@yahoo.com.br

\section{RESUMO}

O objetivo do presente trabalho foi avaliar o efeito da inoculação de Bacillus subtilis e Azospirillum brasilense no crescimento e na produção de milho. O experimento foi conduzido no município de São Jorge do Ivaí, durante o período de abril a agosto de 2013, em delineado no esquema de blocos casualizados, com cinco tratamentos e quatro repetições. T1 - Controle; T2 - Bacillus subtilis em suspensão aquosa $\left(1 \mathrm{~L} \mathrm{ha}^{-1}\right)$ no sulco de semeadura; T3-Bacillus subtilis em pó (1 kg ha${ }^{1}$ ) inoculado nas sementes; T4 - Azospirillum (100 $\mathrm{mL} \mathrm{ha}^{-1}$ ) inoculado nas sementes; T5 Azospirillum $\left(100 \mathrm{~mL} \mathrm{ha}^{-1}\right)+$ Bacillus $\left(1 \mathrm{~L} \mathrm{ha}^{-1}\right)$ no sulco. Foram realizadas análises de nematoides nas raízes do milho, massa fresca da parte aérea, peso da espiga e altura de plantas e avaliado o rendimento da cultura. Foi utilizado o programa Sisvar para realização da analise estatística. Para análise de variância foi utilizado o teste $\mathrm{F}$ e Tukey (5\%) para comparação das médias. Não houve controle populacional dos fitonematoides avaliados durante a condução do experimento. A utilização de Bacillus subtilis possibilitou um desenvolvimento da massa fresca da parte aérea em aproximadamente $15 \%$ do milho no cultivo safrinha. O uso de Azospirillum brasilense nas sementes aumentou em $21,9 \%$ a produtividade do milho, quando comparados ao tratamento controle.

Palavras-chave: rizobactérias; produtividade; Zea mays.

INOCULATION OF Bacillus subtilis AND Azospirillum brasilense ON GROWTH AND YIELD OF CORN

\begin{abstract}
The objective of this study was to evaluate the effect of inoculation of Bacillus subtilis and Azospirillum brasilense on growth and yield of corn. The experiment was conducted in the municipality of São Jorge Ivaí during the period April to August 2013 in outlined in a randomized block design with five treatments and four replications. T1 - Control; T2 - Bacillus subtilis suspended in water (1 L ha-1) at sowing; T3 - Bacillus subtilis powder ( $\left.1 \mathrm{~kg} \mathrm{ha}^{-1}\right)$ inoculated seeds; T4 - Azospirillum (100 mL ha-1) inoculated seeds; T5 - Azospirillum (100 mL ha $\left.{ }^{-1}\right)+$ Bacillus (1 L ha-1) in the groove. Analysis of nematodes in the roots of corn, fresh weight of shoots, ear weight and plant height were performed and evaluated crop yield. Sisvar the program to conduct statistical analysis was used. For the analysis of variance $F$ and Tukey test (5\%) for comparison of means was used. There was no population control of plant nematodes evaluated during the experiment. The use of Bacillus subtilis has supported development of the fresh weight of shoots in approximately $15 \%$ of corn in the off-season cultivation. The use of Azospirillum brasilense in the seeds increased by $21.9 \%$ corn yield compared to the control treatment.
\end{abstract}

Keywords: rhizobacteria; productivity; Zea mays. 


\section{INTRODUÇÃO}

Em função do potencial produtivo do milho, a sua composição química e valor nutritivo constituem-se em um dos mais importantes cereais cultivados e consumidos no mundo. Devido a sua multiplicidade e aplicações, na alimentação humana ou na alimentação animal, assume relevante papel socioeconômico, além de constituir-se em indispensável matéria-prima, impulsionadas de diversificados complexos agroindustriais (FANCELLI; DOURADO NETO, 2000).

A utilização do milho em grão como alimentação animal representa cerca de $70 \%$ em todo o mundo. Nos Estados Unidos 50\% são destinados para essa finalidade, enquanto no Brasil o seu percentual varia entre 60 a 80\% (GODOY, 2002). Após o trigo e do arroz, o milho (Zea mays L.) é o cereal mais cultivado no mundo (FAO, 2008).

$\mathrm{O}$ crescimento e a produtividade do milho são em função do potencial genético para reagir às condições ambientais as quais ele está submetido. O ambiente fornece a maior influência sobre estes fatores, mas o produtor pode utilizar práticas eficientes para a obtenção de maiores produtividades, aumentando consequentemente $\mathrm{o}$ lucro (RITCHIE et al., 2003). Há necessidade de sementes de milho de alta qualidade, já que a base da alta produção está diretamente relacionada com o estabelecimento das plantas no campo (MACHADO et al., 2001).
O milho é uma cultura que está presente em todas as regiões brasileiras, nos últimos anos, os custos de produção aumentaram. As utilizações de técnicas que proporcionem economia, sem perda de produtividade agrícola, podem incentivar seu cultivo que é de grande importância no país para pequenos e grandes produtores (SILVA; SOUZA, 2007).

A inoculação com Azospirillum amazonense no cultivo do milho promove a maior produção de matéria seca e acúmulo de nitrogênio nas raízes, é uma bactéria promotora do crescimento de plantas, realiza a fixação biológica do nitrogênio, indicando desta forma sua inoculação, na cultura do milho e outras gramíneas (REIS JUNIOR et al., 2008). Devem ser considerados os benefícios por menor poluição ambiental que resulta da produção e utilização de fertilizantes nitrogenados, bem como pela redução na emissão de gases de efeito estufa. Desse modo, além da economia para os agricultores, o uso de inoculantes contendo Azospirillum contribui para o ambiente e pode ser objeto de negociações futuras no comércio de créditos de carbono (CORREA et al., 2008).

Nematoides do gênero Meloidogyne acarretam grandes danos ao sistema radicular das plantas. Estes parasitas injetam toxinas e também provocam deformações conhecidas como "galhas", acarretando, 
enfim em baixo desenvolvimento e pouca eficiência das raízes, consequentemente ocorrem reduções na produtividade (DINARDO-MIRANDA, 2005). Os nematoides do gênero Pratylenchus são conhecidos como nematoides das lesões radiculares. Estes parasitas penetram e saem livremente das raízes, já que não há nenhuma fase ou estágio que possa ser denominado infestante. Este parasitismo acarreta em aberturas nas raízes, onde podem penetrar bactérias e fungos patogênicos. Os nematoides deste gênero abandonam o sistema radicular quando encontram condições desfavoráveis, migrando para o solo (GOULART, 2008).

O Bacillus subtilis, é uma bactéria habitante natural do solo, que produz antibióticos, enzimas e fito hormônios que vai proporcionar benefícios para as plantas. É uma espécie microbiana, também descrita como rizobactéria promotora de crescimento de plantas. A rizobactéria promotora de crescimento de plantas é um agentes potencial para controle biológico de fitopatógenos, como por exemplo, os nematoides (KLOEPPER et al., 1999). Essas rizobactérias podem acabar ou controlar as doenças por modos de ação: por antagonismo relacionado à produção de antibióticos antifúngicos como iturina em $B$. subtilis (ARAÚJO et al., 2005).
O objetivo do presente trabalho foi avaliar o efeito da inoculação de Bacillus subtilis e Azospirillum brasilense no crescimento e na produção de milho safrinha no norte do Paraná.

\section{METODOLOGIA}

O experimento foi conduzido no município de São Jorge do Ivaí, latitude 23으 $25^{\prime} 58^{\prime \prime} \mathrm{S}$, longitude 52 $17^{\prime} 35^{\prime \prime} \mathrm{W}$, altitude $430 \mathrm{~m}$, no noroeste do Paraná, durante o período de abril a agosto de 2013 (cultivo safrinha). O clima da região climática classificada, segundo o modelo de Köppen, como do tipo Cfa. O solo é classificado como Latossolo Roxo eutrófico com A moderado textura argilosa, e o relevo suave ondulado, apresentando o seguinte perfil de fertilidade (RAIJ et al., 2001): $\mathrm{pH} \mathrm{CaCl}=5,1 ; \mathrm{pH}\left(\mathrm{H}_{2} \mathrm{O}\right)=$ 5,8; $\mathrm{pH}(\mathrm{SMP})=6,30 ; \mathrm{H}+\mathrm{Al}=3,1 \mathrm{cmol} ; \mathrm{Al}=0$ ${ }_{c} \mathrm{~mol} ; \mathrm{Ca}=68 \mathrm{mmolc} \mathrm{dm}^{-3} ; \mathrm{Mg}=18 \mathrm{mmolc}^{-}$ 3; $\mathrm{K}=7,5 \mathrm{mmolc} \mathrm{dm}^{-3} ; \mathrm{P}$ (Mehlich) $=11,0 \mathrm{mg}$ $\mathrm{dm}^{-3} ; \mathrm{P}($ Resina $)=21,0 \mathrm{mg} \mathrm{dm}^{-3} ;$ M. O. $=3,3 \mathrm{~g}$ $\mathrm{dm}^{-3} ; \mathrm{SB}=93,5 \mathrm{mmol} ; \mathrm{CTC}=124,5 \mathrm{cmol} ; \mathrm{V} \%$ $=75,10$. O clima da região é classificado segundo o modelo de Köppen, como do tipo Cfa, com precipitação anual média de 1875,4 $\mathrm{mm}$. O experimento foi delineado no esquema de blocos casualizados. As parcelas possuíam $20 \mathrm{~m}$ de comprimento por $5,40 \mathrm{~m}$ de largura, totalizando $108 \mathrm{~m}^{2}$ por parcela, sendo quatro blocos, em cada bloco os 5 
tratamentos se alternavam, a área total conduzida foi de $2160 \mathrm{~m}^{2}$.

Foram definidos os seguintes tratamentos, com quatro repetições:

T1 - Controle;

T2 - Bacillus subtilis em suspensão aquosa (1 $\left.\mathrm{L} \mathrm{ha}^{-1}\right)$ no sulco de semeadura;

T3 - Bacillus subtilis em pó (1 $\left.\mathrm{kg} \mathrm{ha}^{-1}\right)$ inoculado nas sementes;

T4 - Azospirillum (100 mL ha $\left.{ }^{-1}\right)$ inoculado nas sementes;

$\mathrm{T5}$ - Azospirillum (100 $\left.\mathrm{mL} \mathrm{ha}{ }^{-1}\right)+$ Bacillus (1 $\mathrm{La}^{-1}$ ) no sulco de semeadura.

Para inoculação com Bacillus subtilis foi utilizado um produto comercial em pó (FATEC) contendo a concentração de $1,0 \times 10^{8}$ células por grama. Para inoculação do Azospirillum foi utilizado inoculante comercial contendo a concentração de $1,0 \times 10^{9}$ células por $\mathrm{mL}$.

A semeadura do milho foi realizada no dia vinte e três de março de 2013, a semente utilizada foi da cultivar Pionner 3431 Híbrido simples. A semeadura foi feita com uma semeadora de seis linhas, com espaçamento entre linhas de noventa centímetros, sendo regulada para distribuir 5,2 sementes por metro linear, com adubação na base com formulação 08-20-20 com a dose de $300 \mathrm{~kg}$ por hectare. Na semeadora havia acoplado um equipamento para a inoculação direta no sulco de semeadura, sendo utilizado para o tratamento com a aplicação de Bacillus subtilis em suspensão aquosa $\left(1 \mathrm{~L} \mathrm{ha}{ }^{-1}\right)$ e Azospirillum (100 mL ha ${ }^{-1}$ ) com B. subtilis (1L $\mathrm{ha}^{-1}$ ), os dois tratamentos consistiam na diluição do produto em água, na dosagem de $300 \mathrm{~L}$ de volume de calda $\mathrm{ha}^{-1}$.

Após a semeadura foram retiradas amostras de solo, e encaminhadas ao laboratório de Microbiologia da Unoeste para a quantificação de nematoides, encontrando em média 500 nematoides a cada $100 \mathrm{~g}$ de solo. Durante a condução do experimento foram efetuadas avaliações do crescimento das plantas e índice populacional de nematoides. Aos 60 dias foram retiradas amostras de raízes para a quantificação e identificação de nematoides. Aos 120 dias após o plantio foram realizadas análises de massa fresca da parte aérea, peso da espiga e altura de plantas.

No final do ciclo da cultura, aos 137 dias após o plantio, foi avaliado o rendimento considerando a produção de grãos na área útil das parcelas, em 4 linhas espaçadas a 0,9 m, com 20 m de comprimento, totalizando 72 $\mathrm{m}^{2}$.

Os resultados obtidos foram submetidos à análise de variância, através do programa computacional Sistema de Análise de Variâncias - SISVAR (FERREIRA, 2000), sendo as médias comparadas pelo teste Tukey. 


\section{RESULTADOS}

A análise de nematoides nas raízes do milho indicou que os tratamentos efetuados com as rizobactérias não mostraram diferenças significativas na população de nenhum dos gêneros de nematoides presentes na área do cultivo (Tabela 1).

Tabela 1. Nematoides dos gêneros Meloidogyne e Pratylenchus em $20 \mathrm{~g}$ de raízes, com inoculação de Bacillus subtilis e Azospirillum brasilense na semente e no sulco de semeadura para o cultivo de milho.

\begin{tabular}{lccc}
\hline Tratamentos & Meloidogyne & Pratylenchus & Ovos \\
\hline Bacillus subtilis no sulco & $160,0 \mathrm{a}$ & $80,0 \mathrm{a}$ & $160,0 \mathrm{a}$ \\
Bacillus subtilis na semente & $120,0 \mathrm{a}$ & $80,0 \mathrm{a}$ & $160,0 \mathrm{a}$ \\
Azospirillum brasilense na semente & $160,0 \mathrm{a}$ & $120,0 \mathrm{a}$ & $160,0 \mathrm{a}$ \\
Azospirillum com Bacillus subtilis & $160,0 \mathrm{a}$ & $80,0 \mathrm{a}$ & $80,0 \mathrm{a}$ \\
Controle & $320,0 \mathrm{a}$ & $80,0 \mathrm{a}$ & $200,0 \mathrm{a}$ \\
\hline C.V(\%) & 30,74 & 28,75 & 37,22 \\
\hline
\end{tabular}

Médias seguidas de mesma letra não diferem estatisticamente pelo teste Tukey (5\%)

A análise da altura das plantas de milho indicou um maior desenvolvimento das plantas com a realização do tratamento de co-inoculação de Azospirillum brasilense com Bacillus subtilis, enquanto os demais tratamentos não diferiram significativamente do controle (Tabela 2).

A massa fresca da parte aérea das plantas de milho evidenciou que 0 tratamento com Bacillus subtilis aplicado sulco de semeadura apresentou o maior desenvolvimento das plantas em relação aos outros tratamentos, sendo $14,87 \%$ superior ao tratamento controle. A co-inoculação de
Azospirillum com Bacillus subtilis e o tratamento controle apresentaram as menores médias da massa fresca da parte aérea das plantas de milho (Tabela 2 ).

A massa das espigas de milho foi maior no tratamento com Bacillus subtilis aplicado nas sementes, sendo 15,34\% superior ao tratamento controle, que apresentou os menores valores de peso de espiga (Tabela 2). 
Tabela 2. Altura das plantas em metro, aos 120 dias após o plantio, com inoculação de Bacillus subtilis e Azospirillum brasilense na semente e no sulco de semeadura para o cultivo de milho.

\begin{tabular}{lccc}
\hline Tratamentos & Altura - $\mathrm{m}$ & MFPA - kg & Peso da espiga - kg \\
\hline Bacillus subtilis no sulco & $1,91 \mathrm{~b}$ & $0,518 \mathrm{a}$ & $0,410 \mathrm{ab}$ \\
Bacillus subtilis na semente & $1,91 \mathrm{~b}$ & $0,503 \mathrm{ab}$ & $0,417 \mathrm{a}$ \\
Azospirillum brasilense na semente & $1,92 \mathrm{ab}$ & $0,476 \mathrm{bc}$ & $0,379 \mathrm{c}$ \\
Azospirillum com Bacillus subtilis & $1,97 \mathrm{a}$ & $0,450 \mathrm{c}$ & $0,396 \mathrm{bc}$ \\
Controle & $1,88 \mathrm{~b}$ & $0,441 \mathrm{c}$ & $0,353 \mathrm{~d}$ \\
\hline C.V(\%) & 3,03 & 9,37 & 5,94 \\
\hline
\end{tabular}

Médias seguidas de mesma letra não diferem estatisticamente pelo teste Tukey (1\%).

A produtividade do milho foi maior no tratamento com a inoculação de Azospirillum brasilense nas sementes, aumentando a produtividade do milho em cerca de 12 sacas por hectare, uma superioridade de $21,9 \%$ em relação ao tratamento controle (Tabela3).

Tabela 3. Produtividade em quilograma por hectare, com inoculação de Bacillus subtilis e Azospirillum brasilense na semente e no sulco de semeadura para o cultivo de milho.

\begin{tabular}{lc}
\hline Tratamentos & Produtividade \\
\hline Bacillus subtilis sulco & $3166,30 \mathrm{ab}$ \\
Bacillus subtilis semente & $3077,48 \mathrm{ab}$ \\
Azospirillum brasilense semente & $3364,03 \mathrm{a}$ \\
Azospirillum com Bacillus subtilis & $3146,19 \mathrm{ab}$ \\
Controle & $2626,17 \mathrm{~b}$ \\
\hline C.V(\%) & 10,47 \\
\hline
\end{tabular}

Médias seguidas de mesma letra não diferem estatisticamente pelo teste Tukey (5\%).

\section{DISCUSSÃO}

Freitas (2001) afirma que rizobactérias podem produzir substâncias tóxicas ou repelentes que podem desestimular a penetração ou alimentação de nematoides nas raízes das plantas. Conforme constatado por Silveira (2001), as rizobactérias podem atuar na infectividade, virulência e agressividade do patógeno, e também nos processos de infecção, desenvolvimento de sintomas e reprodução. Entretanto, as rizobactérias avaliadas no experimento não contribuíram para controlar de maneira significativa a infestação de nematoides nas raízes do milho.

A utilização de rizobactérias promotoras de crescimento em plantas, promovem uma colonização das raízes e estimulam diretamente beneficiamento 0 crescimento e desenvolvimento de diversas plantas (ROESCH et al., 2007).

Araújo et al. (2009) constatou um maior crescimento da parte aérea de tomate tratado com Bacillus subtilis, o que 
caracteriza a bactéria como promotora de crescimento de planta, e esse efeito pode ser devido, à produção de fitoreguladores vegetais por $B$. subtilis na rizosfera das plantas (ARAÚJO et al., 2005).

Araújo (2008) avaliando a inoculação de Bacillus subtilis no milho, conclui que o Bacillus subtilis formulado com a farinha de ostras (BSFO) e inoculado nas sementes de milho apresenta potencial para incrementar o crescimento e a nutrição das plantas. Lima et al. (2011) constatou que a inoculação das sementes com Bacillus subtilis melhorou o desenvolvimento e aumentou a produtividade de grãos do milho.

A aplicação de rizobactérias promotoras de crescimento, promovendo benefícios para a agricultura, pois possuem potencial para estimular crescimento, inibição de fitopatógenos, promover a disponibilização de nutrientes, tornando a planta mais resistente a estresses bióticos e abióticos. Estas bactérias quando aplicadas ao solo proporcionam uma produção mais sustentável, com economia de fertilizantes (ARAÚJO, 2008).

\section{CONCLUSÃO}

Não houve controle populacional dos fitonematoides avaliados durante a condução do experimento.

A utilização de Bacillus subtilis possibilitou um desenvolvimento da massa fresca da parte aérea em aproximadamente $15 \%$ do milho no cultivo safrinha. O uso de Azospirillum brasilense nas sementes aumentou em $21,9 \%$ a produtividade do milho, quando comparados ao tratamento controle.

\section{REFERÊNCIAS}

ARAÚJO, F. F.; MARCHESI, G. V. P. Uso de Bacillus subtilis no controle da meloidoginose e na promoção do crescimento do tomateiro. Ciência Rural, vol.39, n.5, p. 1558-1561. 2009. http://dx.doi.org/10.1590/S0103$\underline{84782009000500039}$

ARAUJO, F. F.; HENNING, A.; HUNGRIA, M. Phytohormones and antibiotics produced by Bacillus subtilis and their effects on seed pathogenic fungi and on soybean root development. World Journal of Microbiology \& Biotechnology, Dordrecht, v. 21, p. 16391645, 2005. http://dx.doi.org/10.1007/s11274-005-3621$\underline{x}$

ARAÚJO, F. F. Inoculação de sementes com Bacillus subtilis, formulado com farinha de ostra e desenvolvimento de milho, soja e algodão. Ciência e agrotecnologia, Lavras, v.2, p. 456-462, 2008.

CORREA, O.S.; ROMERO, A.M.; SORIA, M.A.; DE ESTRADA, M. Azospirillum brasilense plant genotype interactions modify tomato response to bacterial diseases, and root and foliar microbial communities. In: CASSÁN, F.D.; GARCIA DE SALAMONE, I. (Ed.)

Azospirillum sp.: cell physiology, plant interactions and agronomic research in Argentina. Argentina: Asociación Argentina de Microbiologia, 2008. p.87-95.

DINARDO-MIRANDA, L. L. Nematoides e pragas de solo em cana-de-açúcar. Potafos 
Informações Agronômicas, n.110, p.25-32, 2005.

FANCELLI, A.L.; DOURADO NETO, D. Produção de milho. Guaíba: Agropecuária, 360p. 2000.

FAO. Food and Agriculture Organization of the United Nations. The state of food and agriculture. Rome: FAO, 2008.

FERREIRA, D. F. Análise estatística por meio do SISVAR (Sistema para Análise de Variância) para Windows versão 4.0. In: REUNIÃO ANUAL DA REGIÃO BRASILEIRA DA SOCIEDADE INTERNACIONAL DE BIOMETRIA, 45., 2000, São Carlos. Anais... São Carlos: UFSCar, p. 255-258. 2000.

FREITAS, L.G. Rizobactérias versus nematoides. 2001. Disponível em: <http://www.ufv.br/dpf/labnematologia/rizo .pdf>. Acesso em: 05 jul. 2014.

GODOY, L. J. G. Manejo do nitrogênio em cobertura na cultura do milho (Zea mays L.) em solo arenoso baseado no índice relativo de clorofila. 94p. Dissertação (Mestrado em Agricultura) - Faculdade de Ciências Agronômicas, Universidade Estadual Paulista, Botucatu, 2002.

GOULART, A. M. C. Aspectos gerais sobre nematoides das lesões radiculares (gênero Pratylenchus). Planaltina: Embrapa, 2008. 27p. (Documentos, n. 219).

KLOEPPER, J.W.; LIFSHITZ, R.; ZABLOTOWICZ, R.M. Free-living bac- terial inocula for enhancing crop productivity. Trends in Biotechnology, v.7, p.39-43, 1999. http://dx.doi.org/10.1016/01677799(89)90057-7

LIMA, F.F.; NUNES, L.A.P.L.; MARCIA DO V. B. FIGUEIREDO, M.V.B.; ARAÚJO, F.F.; LUCIANO M. LIMA, L.M.; ARAÚJO, A.S.F. Bacillus subtilis e adubação nitrogenada na produtividade do milho. Revista Brasileira de Ciências Agrárias, v. 6, 2011.

MACHADO, J. C.; OLIVEIRA, J. A.; VIEIRA, M. G. G. C.; ALVES, M. C. Uso da restrição hídrica na inoculação de fungos em sementes de milho. Revista Brasileira de Sementes, v. 23, n. 2, p.88-94, 2001.

RAIJ, B. V.; ANDRADE, J. C.; ANTARELLA, H.; QUAGGIO, J. A. Análise química para avaliação da fertilidade de solos tropicais. Campinas: Instituto Agronômico, 2001.

REIS JUNIOR, F. B. et al. Inoculação de Azospirillum amazonense em dois genótipos de milho sob diferentes regimes de nitrogênio. Revista Brasileira de Ciência do solo, Brasília, v. 32, p. 1139-1146, 2008.

RITCHIE, S. W.; HANWAY, J. J.; BENSON, G. O. Como a planta de milho se desenvolve. Piracicaba: Potafos, 2003. Informações Agronômicas, n.103.

ROESCH, L. F. W.; PASSAGLIA, L. M. P.; BENTO, F. M.; TRIPLETT, E. W.; CAMARGO, F. A. O. Diversidade de bactérias diazotróficas endofíticas associadas a plantas de milho. Revista Brasileira de Ciências do Solo, v.31, n.1367-1380, 2007.

SILVA, D. A.; SOUZA, L. C. F. Análise econômica de sucessões de culturas para milho, com níveis de nitrogênio em cobertura. Revista Brasileira de Milho e Sorgo, v.6, n.2, p. 256-262, 2007.

SILVEIRA, E.B. Bactérias promotoras de crescimento de plantas e biocontrole de doenças. In: MICHEREFF, S.J.; BARROS, R. Proteção de plantas na agricultura sustentável. Recife: UFRPE, 2001. p.70-100. 